\title{
Liposarcome cervical géant exteriorisé et radio-induit : à propos d'une observation
}

\section{Geant liposarcoma of the neck, exteriorized and radiation-induced: a case report}

\author{
Y. Yazibene $\cdot$ N. Ait-mesbah $\cdot$ M. Benada $\cdot$ N. Yahi-aitmesbah $\cdot$ M. Mokhtari $\cdot$ K. Messaoudene $\cdot$ W. Touisi $\cdot$ K. Kalem \\ Reçu le 17 février 2012 ; accepté le 6 mars 2012 \\ (C) Springer-Verlag France 2012
}

Résumé Objectif : Étude des caractéristiques cliniques, radiologiques, et évolutives avec une revue de littérature d'une tumeur cervicale, hautement maligne, peu fréquente et radio-induite (liposarcome).

Patient et méthodes : Nous rapportons le cas d'un patient âgé de 38 ans, aux antécédents de néoplasie du cavum (UCNT) qui remonte à 13 ans, traité par une radiothérapie à dose curatrice et une chimiothérapie adjuvante et présentant une tuméfaction cervicale d'allure maligne par son aspect macroscopique et sa croissance rapide. À titre d'exploration, un scanner cervical effectué avec un bilan d'extension et une exérèse chirurgicale a été le principal traitement.

Résultats : La TDM objective une masse latérocervicale droite évoquant plusieurs étiologies (tumeur mésenchymateuse, adénopathies cervicales remaniées ?). L'histologie de la biopsie révèle un liposarcome atypique. Le bilan d'extension est revenu normal.

Nous avons pratiqué une exérèse chirurgicale incomplète en raison de l'extension basicrânienne. L'histologie de la pièce opératoire révèle un liposarcome dédifférencié.

La récidive est survenue huit mois après.

Conclusion : Le LSRI est une complication rare et tardive d'un traitement ionisant. Son agressivité dépend essentiellement de la forme histologique. Il pose un problème de prise en charge.

Le traitement est fondé sur l'ablation chirurgicale.

Mots clés Liposarcome extériorisé · Radio-induit .

Atypique $\cdot$ Terrain irradié $\cdot$ Récidive locale

\footnotetext{
Y. Yazibene $(\bowtie) \cdot$ N. Ait-mesbah $\cdot$ M. Benada $\cdot$

N. Yahi-aitmesbah

Service ORL et CCF, EPH de Kouba, Alger, Algérie

e-mail : yyazibene@yahoo.fr

M. Mokhtari $\cdot$ K. Messaoudene

Service d'anesthésie-réanimation, EPH de Kouba, Alger, Algérie

W. Touisi $\cdot$ K. Kalem

Service d'histopathologie, hôpital Parnet, Alger, Algérie
}

\begin{abstract}
Objective: Study of clinical, radiological and scalable characteristcs of a cervical tumor (liposarcoma), highly malignant, rare and radiation-induced.

Patient and methods: We report the case of a 38 years old patient, with naso-pharynx neoplasy antecedents (UCNT) since 13 years, treated by a curative radiotherapy and an auxiliary chemotherapy, and who has an atypical swelling of malignant pace by its macroscopic aspect and its rapid growth. As exploration, a CT of the neck carried out with an assessment of extension and a surgical removing was the basic treatment.

Results: The CT objectifies a right mass of the neck evoking several etiologies (mesenchymatous tumor, malignant metastatic gonglion ?). The biopsy reveals an atypical liposarcoma, and the assessment of extension returned normaly.

We practiced a surgical removing. The histology of the operational part reveals a dedifferenciated liposarcoma.

Ablation was not total because of the extension of the tumor to the level of the skull base. Recurrence occurred eight months after.

Conclusion: The LSRI is a rare and late complication of an ionizing treatment. Its aggressiveness depends primarily on the histological form. The treatment is based on surgical ablation.
\end{abstract}

Keywords Exteriorized liposarcoma $\cdot$ Radiation-induced Atypical $\cdot$ Irradiated ground $\cdot$ Local reccurence

\section{Introduction}

Les sarcomes représentent moins de $1 \%$ des néoplasmes malins de la tête et du cou, leur croissance et leur degré d'agressivité varie en fonction du type histologique, son extension est généralement locale.

Leur étiologie inclut le potentiel tumorogénique de la radiothérapie. La nature lipomateuse est encore plus rare au 
niveau cervical, il est classiquement décrit dans l'espace retro péritonéal et les extrémités [1].

\section{Cas clinique}

Il s'agit d'un patient âgé de 38 ans, aux antécédents de cancer du cavum qui remonte à 13 ans, classé T2N2MO, traité par radiothérapie externe (télécobaltothérapie) à raison de 70 GYS, associé à une chimiothérapie adjuvante, avec une rémission clinique complète.

Il nous a été adressé par son médecin radiothérapeute pour une tuméfaction latérocervicale droite apparue récemment (Fig. 1).

L'examen retrouve une tuméfaction latérocervicale droite, composée de deux formes complètement distinctes, l'une est mastoïdienne de $10 \mathrm{~cm}$ de grand diamètre, indolore, multilobée, fixée aux plans profonds avec une peau en regard amincie et luisante, l'autre est basicervicale bourgeonnante et extériorisée de $15 \mathrm{~cm}$ de diamètre d'aspect framboisée et saignante au contact (Fig. 1).

L'endoscopie nasale retrouve un cavum scléreux, sans signes de récidive locale. Le reste de l'examen est sans particularités, et le bilan d'extension est revenu normal.

La TDM réalisée avant l'extériorisation de la masse, objective une formation pseudokystique cloisonnée, fixant le produit de contraste à limites floues, se développant le long du muscle sternocléidomastoïdien, avec une grosse voussure cutanée en regard, faisant évoquer une métastase ganglionnaire probable ou une tumeur mésenchymateuse.

Nous avons pratiqué une cervicotomie exploratrice, au cours laquelle nous découvrons une masse de consistance

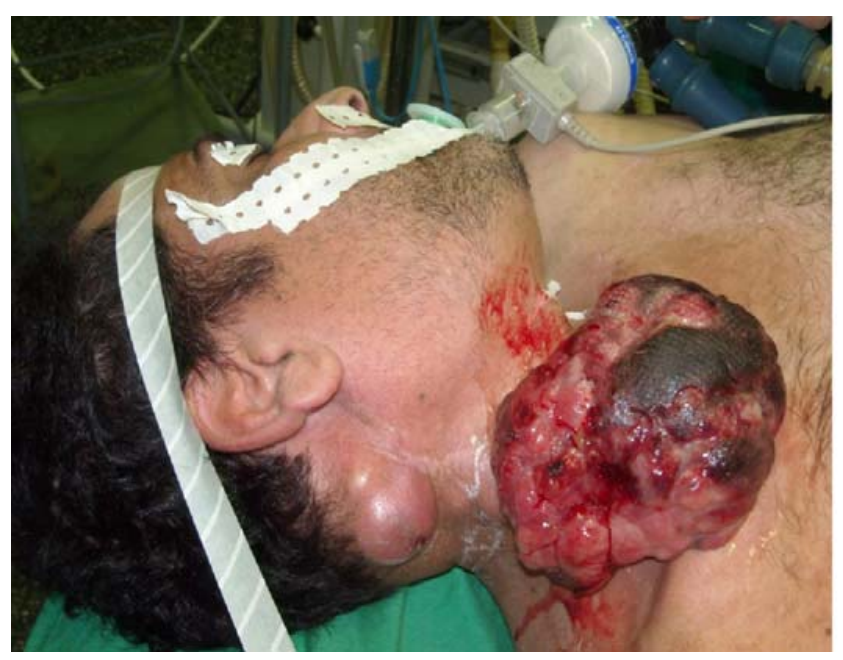

Fig. 1 Deux tuméfactions cervicales distinctes : une basicervicale extériorisée de $15 \mathrm{~cm}$ d'aspect framboisée, saignante au moindre contacte et l'autre haute polylobée retromastoïdienne et occipitale, avec une peau en regard mince et luisante molle, gélatineuse, occupant l'axe vasculaire du cou, adhérente au muscle SCM et étendue à la base du crâne (trou déchiré postérieur), l'exérèse au niveau de cette région n'était pas complète (risque majeur de blessure de la carotide interne et les nerfs mixtes). Une résection cutanée est effectuée avec une reconstruction par un lambeau de rotation (Fig. 2).

Le résultat de l'examen de la pièce opératoire révèle un liposarcome atypique (dédifférencié).

Nous avons adressé le patient au service de radiothérapie pour complément thérapeutique.

À 8 mois de recul, le patient nous a été réadressé pour récidive locale de la masse, devenue très volumineuse, mesurant $30 \mathrm{~cm}$ de diamètre environ (Fig. 3).
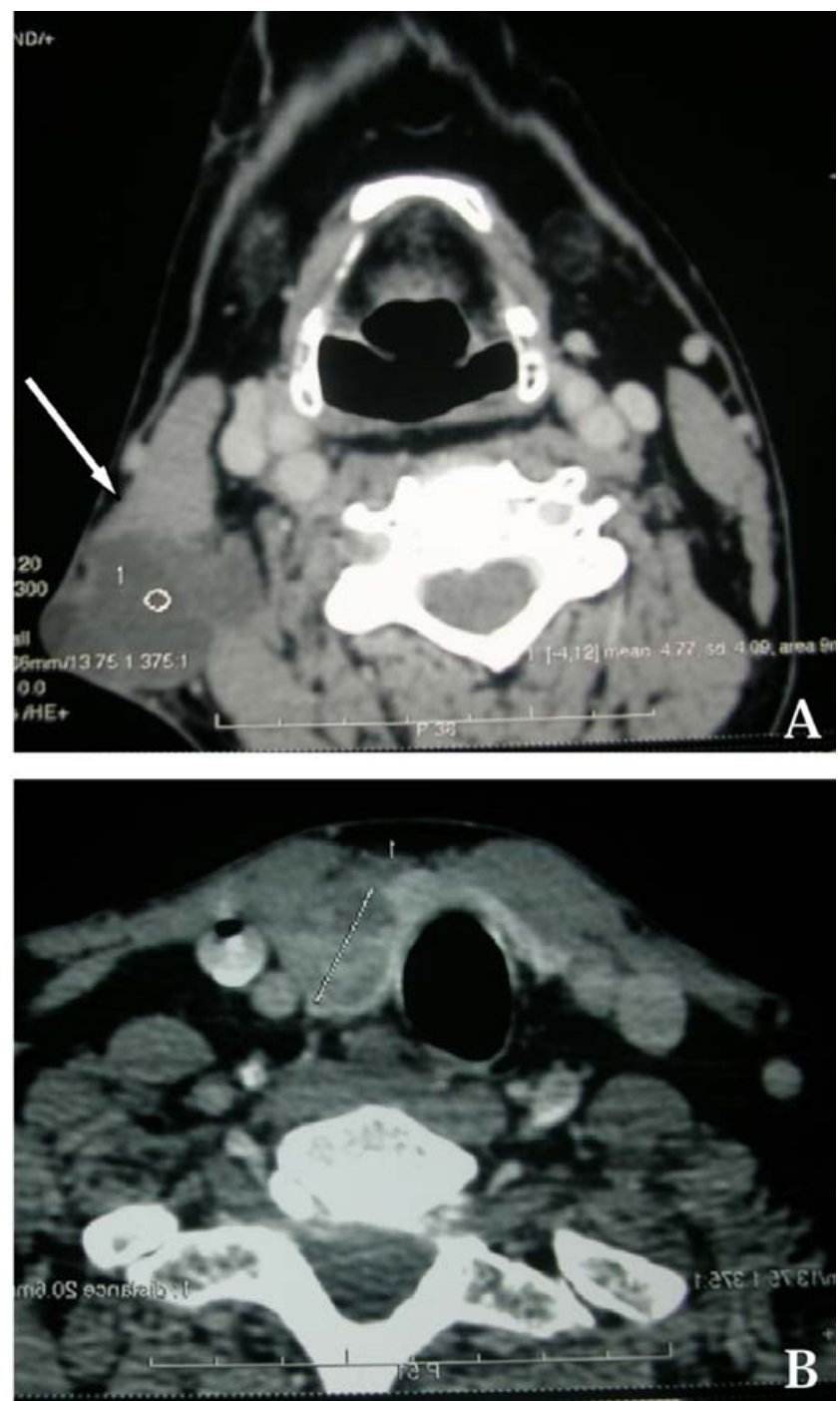

Fig. 2 A : TDM en coupe axiale : image de densité graisseuse, polylobée et cloisonnée, à limites confuses avec le muscle SCM (flèche)

B : TDM en coupe axiale : extension basicervicale de la masse, rehaussée après injection de produit de contraste 

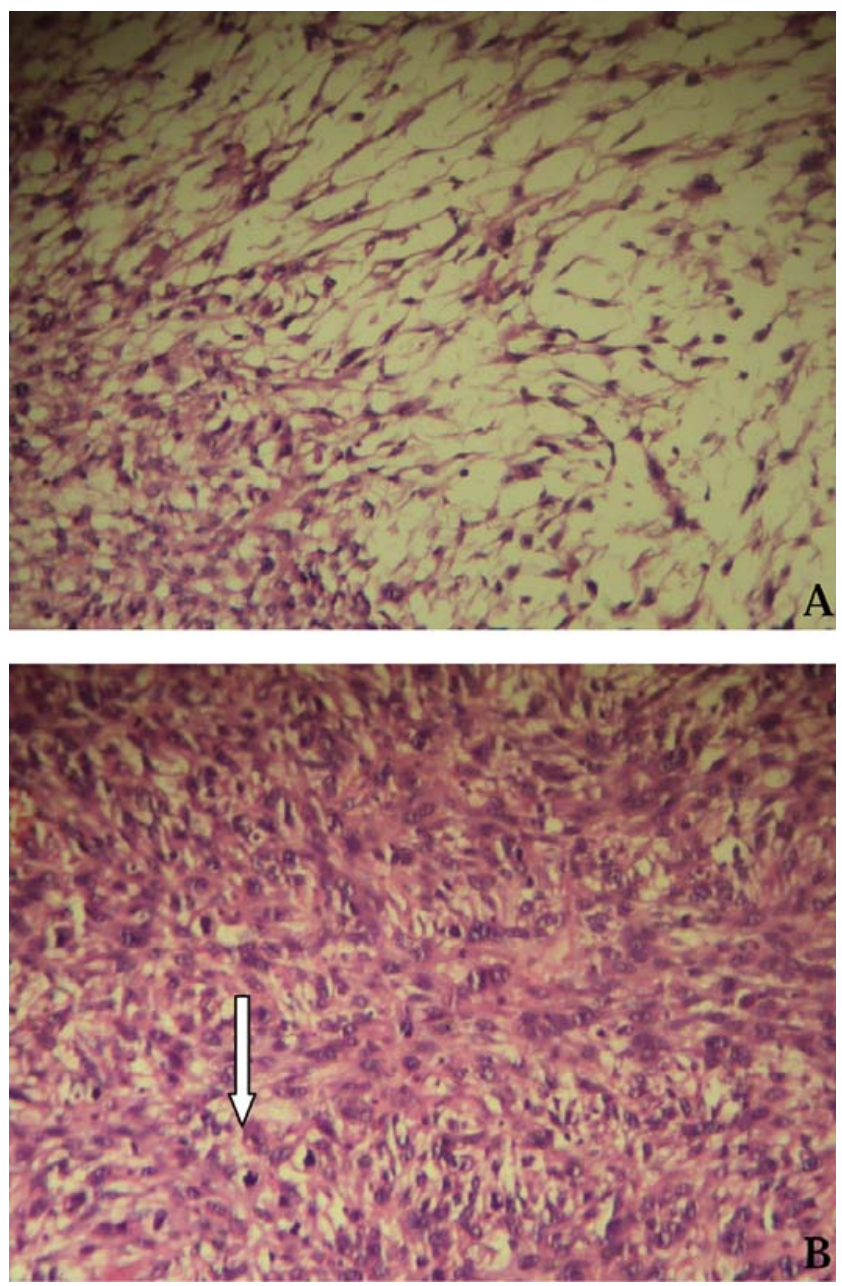

Fig. 3 A : aspect histologique : coloration HE, grossissement $\times$ 20 : liposarcome dédifférencié : prolifération tumorale à double composante, lipomateuse différenciée à droite de l'image, et une zone sarcomateuse indifférenciée à gauche, avec une zone de transition nette (flèche)

B : coloration HE, grossissement x 20 : coupe passant par la zone sarcomateuse. Noter le polymorphisme, la cellularité et les anomalies cytonucléaires

Une chirurgie de réduction tumorale a été tenté. En postopératoire le patient avait présenté des troubles hémodynamiques majeurs nécessitant son transfert au service de réanimation et décède 10 jours après dans un tableau de septicémie.

\section{Discussion}

Les liposarcomes représentent moins de $1 \%$ des néoplasmes malins de la tête et du cou et 10 à $15 \%$ des tous les sarcomes chez l'adulte. Ils se développent à partir des tissus graisseux sous cutané et le tissu conjonctif des organes profonds (espace retropéritonéal), ou il forme la variété la plus commune. Ils affectent essentiellement l'adulte âgé de 40 à 60 ans de sexe masculin (sex ratio de 3/2) [1-3].

Les facteurs étiologiques les plus connus sont la prédisposition génétique, les traumatismes, mais surtout les antécédents de radiothérapie externe qui reste largement discuté dans les différentes publications. Patel et al. [4] ont publié les critères permettant de retenir la notion d'un cancer radioinduit et qui sont dans l'ordre :

1. l'existence d'une période de latence entre la radiothérapie et le diagnostic du cancer, qui doit être supérieure à 5 ans.

2. La confirmation histologique d'un cancer malin développé sur un terrain irradié.

3. La tumeur doit être histologiquement différente de la tumeur traitée initialement.

4. Histoire documentée de l'irradiation du site.

Tous ces critères ont été retrouvés chez notre patient, avec une latence de 13 ans.

Du point de vue histologique, le liposarcome (LS) se développe a partir de 4 types de cellules adipeuses immatures, définissant ainsi 4 sous types. Le LS a cellules ovoïdes à partir des lipoblastes, le LS myxoide à partir des préadipocytes, le LS pléomorphe contenant les différentes variétés suscitées, le LS bien différencié et les LS atypiques, dont fait partie le LS dédifférencié ; celui-ci est formé d'une composante lipomateuse différenciée et d'une autre sarcomateuse indifférenciée. Le degré de malignité dépend du degré de sa différenciation. Macroscopiquement, il s'agit des tumeurs solides en général, avec une pseudocapsule périphérique $[1,5]$. Chez notre patient, c'est plutôt une masse mollasse, gélatineuse à la coupe, avec des zones de nécroses et absence de capsule périphérique.

La revue de la littérature note aussi la taille tumorale qui se situe entre 5 et $10 \mathrm{~cm}[2,3]$.

L'extériorisation externe est une particularité clinique de la tumeur chez notre patient (Figs 1,4), l'évolution a été remarquablement rapide.

Le diagnostic des LSRI repose sur un faisceau d'arguments anamnestiques (antécédents d'irradiation), cliniques, radiologiques et histologique.

La TDM et l'IRM montre la présence de cloisonnements de la masse de densité adipeuse, lui donnant un aspect hétérogène avec des limites peu nettes en général $[5,6]$.

D'autres étiologies peuvent être évoquées, notamment sur le plan radiologique. Ce sont les tumeurs des parties molles incluant l'histiocytome malin, le rhabdomyosarcome, le leiomyosarcome et les tumeurs nerveuses malignes.

L'exérèse chirurgicale large avec ou sans radiothérapie complémentaire constitue l'arme thérapeutique la plus efficace $[7,8]$; celle-ci n'est pas toujours évidente en présence d'une extension vers les organes vitaux de voisinage. La persistance d'une agression tumorale est source d'une 


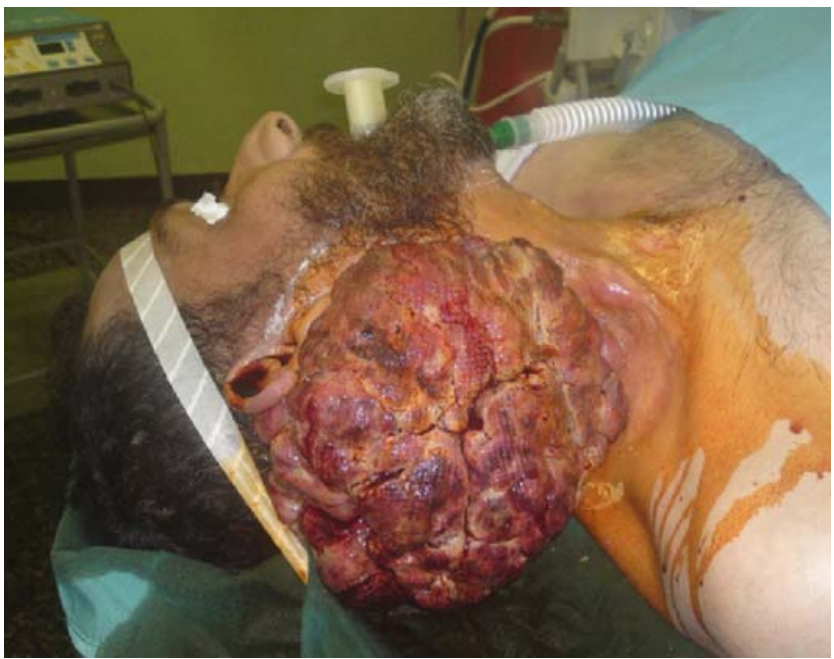

Fig. 4 Récidive tumorale massive huit mois après l'exérèse chirurgicale. Noter l'aspect macroscopique

récidive rapide ( $80 \%$ de récidive à moins de 5 ans). Quand aux métastases à distances, elles sont décrites plus particulièrement pour les formes indifférenciées.

Le pronostic des LSRI est sévère, la survie à 5 ans est de 59 à $64 \%$ pour les formes différenciés et beaucoup plus péjoratif dans les formes indifférenciés (18 à $21 \%$ de survie à 5 ans) $[3,4,7,8]$.

\section{Conclusion}

Les LSRI sont des tumeurs malignes rares au niveau de la tête et du cou, ils sont doués d'un potentiel agressif élevé, avec un fort pourcentage de récurrence locale et de métastases à distance.
L'apparition d'une lésion suspecte sur un terrain irradié doit attirer l'attention du spécialiste, qui doit penser à ces cancers malgré leur rareté. Ces tumeurs posent un véritable problème de prise en charge, parfois insoluble. Le traitement le plus efficace reste l'exérèse chirurgicale la plus large. Celle-ci constitue un véritable challenge pour le chirurgien, qui doit être carcinologiquement valable, toute en respectant les organes vitaux avoisinant.

Conflit d'intérêt : les auteurs ne déclarent pas de conflit d'intérêt.

\section{Références}

1. Coca Pelaz A, Nunez Batalla F, Vivanco Allende B, et al (2010) Giant neck liposarcoma with pathological clavicular fracture. Auris Nasus Larynx 37:394-6

2. Calvo de Mora J, Herencia H, Salmeron JI, et al (2006) Radiationinduced sarcomas of the head and neck. A challenge for the surgeon. J Craniomaxillofac Surg 34(Suppl1):187

3. de Bree R, van der Valk P, Kuik DJ, Paul J, et al (2006) Prognostic factors in adult soft tissue sarcomas of the head and neck: A single-centre experience. Oral Oncology 42:703-9

4. Patel SG, Shaha AR, Shah JP (2001) Soft tissue sarcomas of the head and neck. An update. Am J Otolaryngol 22:2-18

5. Zagers GK, Ballo MT, Pisters PWT, et al (2003) Prognostic factors for patients with localized soft-tissue sarcoma treated with conservation surgery and radiation therapy. Cancer 97:2530-43

6. Shephard D, Libshitz HI (2001) Post-radiation sarcoma: a review of the clinical and imaging features in 63 cases. Clin Radiol 56:22-9

7. Patel SG, See ACH, Williamson PA, Archer DJ (1999) Radiation induced sarcoma of the head and neck. Head Neck 21:346-54

8. Merdan F, Gorkem A (2009) The role of surgery and radiotherapy in treatment of soft tissue sarcomas of the head and neck region: Review of 30 cases. J Craniomaxillofac Surg 37:42-8 